Supporting Information for the paper:

\title{
Transition Metal Migration upon Attempting the Wolff Rearrangement of a Ir(III) Five-Membered Metallacycle
}

Margarita Gómez, Margarita Paneque, ${ }^{*}$ Manuel L. Poveda,* and Eleuterio Alvarez

Instituto de Investigaciones Químicas and Departamento de Química

Inorgánica; Consejo Superior de Investigaciones Cientificas (CSIC) and

Universidad de Sevilla, Av. Américo Vespucio 49, Isla de la Cartuja, 41092

Sevilla, Spain

Synthesis and characterization of compounds $3,4,1,5,6$ and 7 and X-ray structure determination for compounds 5, 6 and 7. 
Compound 3: To a cold $\left(0^{\circ} \mathrm{C}\right)$ solution of $2(0.20 \mathrm{~g}, 0.35 \mathrm{mmol})$ in THF $(10 \mathrm{~mL})$ an excess of $\mathrm{BH}_{3} \cdot \mathrm{THF}(2.5 \mathrm{~mL}, 1 \mathrm{M})$ is added. The reaction mixture is stirred al $0{ }^{\circ} \mathrm{C}$ for 30 min and then at room temperature for $4 \mathrm{~h}$. After cooling the system at $0{ }^{\circ} \mathrm{C}, \mathrm{NaOH}$ (2.5 mL, $3 \mathrm{M}$ in $\mathrm{H}_{2} \mathrm{O}$ ) and $\mathrm{H}_{2} \mathrm{O}_{2}\left(2.5 \mathrm{~mL}, 30 \%\right.$ in $\left.\mathrm{H}_{2} \mathrm{O}\right)$ were added and the resulting mixture stirred for $2 \mathrm{~h}$ at room temperature. Upon addition of $10 \mathrm{~mL}^{\text {of }} \mathrm{Et}_{2} \mathrm{O}$, the organic phase was separated, washed with $\mathrm{NaCl}$-saturated water, dried with $\mathrm{Na}_{2} \mathrm{SO}_{4}$ and taken to dryness. The crude product was crystallized from $\mathrm{Et}_{2} \mathrm{O}: \mathrm{CH}_{2} \mathrm{Cl}_{2}(1: 2)$ at $-20^{\circ} \mathrm{C}$. Isolated yield: $96 \%$.

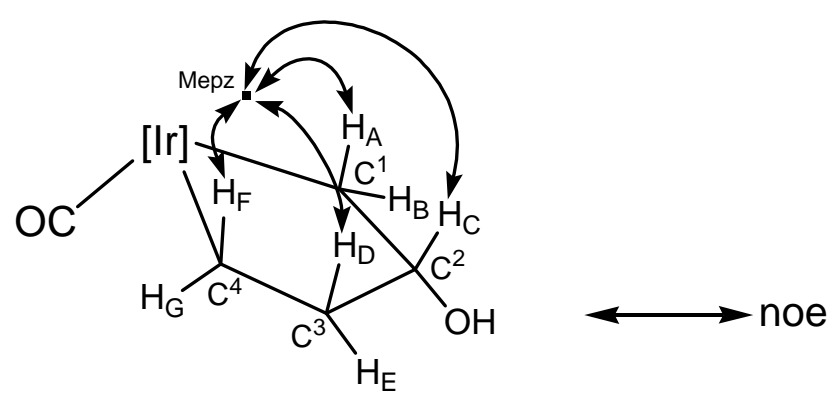

\section{Spectroscopic and analytical data}

IR (Nujol): v $2001 \mathrm{~cm}^{-1}(\mathrm{Ir}-\mathrm{CO})$.

${ }^{1} \mathbf{H}$ NMR $\left(\mathrm{CDCl}_{3}, 25^{\circ} \mathrm{C}\right): \delta 5.78,5.77\left(\mathrm{~s}, 1: 2,3 \mathrm{CH}_{\mathrm{pz}}\right), 4.40\left(\mathrm{~m}, 1 \mathrm{H}, \mathrm{H}_{\mathrm{C}}\right), 2.59(\mathrm{dd}, 1 \mathrm{H}$, $J_{\mathrm{HH}}=12.4$ and $\left.6.6 \mathrm{~Hz}, \mathrm{H}_{\mathrm{D}}\right), 2.50,2.45\left(\mathrm{~m}, 1 \mathrm{H}\right.$ each, $\mathrm{H}_{\mathrm{E}}$ and $\left.\mathrm{H}_{\mathrm{G}}\right), 2.41,2.38,2.36,2.32$ $\left(\mathrm{s}, 1: 1: 1: 3,6 \mathrm{Me}_{\mathrm{pz}}\right), 2.26\left(\mathrm{~m}, 1 \mathrm{H}, \mathrm{H}_{\mathrm{A}}\right), 1.82\left(\mathrm{td}, 1 \mathrm{H}, J_{\mathrm{HH}}=10.2\right.$ and $\left.7.2 \mathrm{~Hz}, \mathrm{H}_{\mathrm{F}}\right), 1.50$ $\left(\mathrm{m}, 1 \mathrm{H}, \mathrm{H}_{\mathrm{B}}\right)$.

${ }^{13} \mathbf{C}\left\{{ }^{1} \mathbf{H}\right\}$ NMR $\left(\mathrm{CDCl}_{3}, 25{ }^{\circ} \mathrm{C}\right): \delta 168.7(\mathrm{CO}), 150.8,150.7,150.1,143.9,143.3$ $\left(1: 1: 1: 1: 2, \mathrm{C}_{\mathrm{qpz}}\right), 109.0,107.0,106.9\left(\mathrm{CH}_{\mathrm{pz}}\right), 80.7\left(\mathrm{C}^{2},{ }^{1} J_{\mathrm{CH}}=141 \mathrm{~Hz}\right), 45.2\left(\mathrm{C}^{3},{ }^{1} J_{\mathrm{CH}}=\right.$ $123 \mathrm{~Hz}), 15.0,14.4,14.2,13.2,12.52,12.5\left(\mathrm{Me}_{\mathrm{pz}}\right), 10.5\left(\mathrm{C}^{1},{ }^{1} J_{\mathrm{CH}}=127 \mathrm{~Hz}\right),-6.1\left(\mathrm{C}^{4}\right.$, ${ }^{1} J_{\mathrm{CH}}=129 \mathrm{~Hz}$ ).

Anal. Calc. for $\mathrm{C}_{20} \mathrm{H}_{30} \mathrm{BN}_{6} \mathrm{O}_{2} \mathrm{Ir}: \mathrm{C}, 40.7 ; \mathrm{H}, 5.1 ; \mathrm{N}, 14.3$. Exp.: C, 40.6; H, 4.8; N, 14.0. 
Compound 4: A solution of $3(0.20 \mathrm{~g}, 0.34 \mathrm{mmol})$ in $\mathrm{CH}_{2} \mathrm{Cl}_{2}(5 \mathrm{~mL})$ is added to a cold $\left(0{ }^{\circ} \mathrm{C}\right)$ suspension of PCC $(0.22 \mathrm{~g}), \mathrm{NaOAc}(0.05 \mathrm{~g})$, celite $(0.6 \mathrm{~g})$ and molecular sieves $(0.1 \mathrm{~g})$ in $\mathrm{CH}_{2} \mathrm{Cl}_{2}(10 \mathrm{~mL})$. After stirring for $30 \mathrm{~min}$ at $0{ }^{\circ} \mathrm{C}$ and for $1 \mathrm{~h}$ at room temperature, the mixture is filtered through a bed of celite and the cake is washed with a little of $\mathrm{CH}_{2} \mathrm{Cl}_{2}$. After removal of the volatiles, compound 4 is crystallized from $\mathrm{Et}_{2} \mathrm{O}: \mathrm{CH}_{2} \mathrm{Cl}_{2}(1: 2)$ at $-20{ }^{\circ} \mathrm{C}$. Isolated yield: $55 \%$.

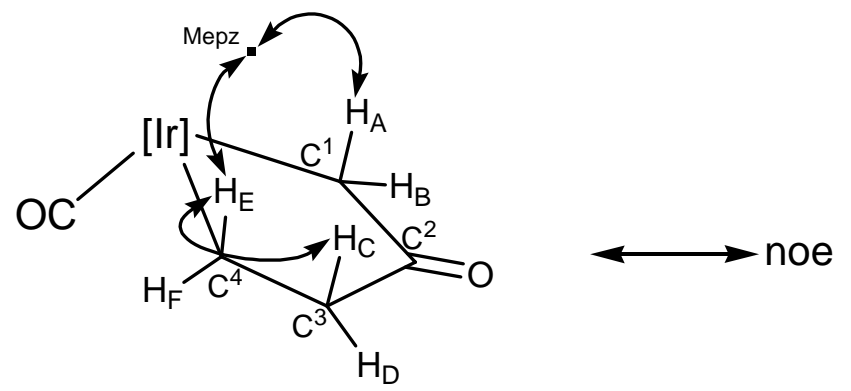

\section{Spectroscopic and analytical data}

IR (Nujol): v $2000(\mathrm{Ir}-\mathrm{CO}), 1696 \mathrm{~cm}^{-1}(\mathrm{C}=\mathrm{O})$.

${ }^{1} \mathbf{H}$ NMR $\left(\mathrm{CDCl}_{3}, 25^{\circ} \mathrm{C}\right): \delta 5.81,5.80,5.79\left(\mathrm{~s}, 1 \mathrm{H}\right.$ each, $\left.3 \mathrm{CH}_{\mathrm{pz}}\right), 3.19\left(\mathrm{~d}, 1 \mathrm{H},{ }^{2} J_{\mathrm{AB}}=\right.$ $\left.17.0 \mathrm{~Hz}, \mathrm{H}_{\mathrm{B}}\right), 2.80\left(\mathrm{dd}, 1 \mathrm{H},{ }^{4} J_{\mathrm{HH}}=1.5 \mathrm{~Hz}, \mathrm{H}_{\mathrm{A}}\right), 2.73\left(\mathrm{dd}, 1 \mathrm{H},{ }^{2} J_{\mathrm{DC}}=17.9,{ }^{3} J_{\mathrm{EC}}=7.3\right.$ $\left.\mathrm{Hz}, \mathrm{H}_{\mathrm{C}}\right), 2.58\left(\mathrm{t}, 1 \mathrm{H},{ }^{2} J_{\mathrm{EF}}={ }^{3} J_{\mathrm{DF}}=10.6 \mathrm{~Hz}, \mathrm{H}_{\mathrm{F}}\right), 2.44\left(\mathrm{dt}, 1 \mathrm{H},{ }^{3} J_{\mathrm{ED}}=10.0 \mathrm{~Hz}, \mathrm{H}_{\mathrm{D}}\right)$, 2.40, 2.34, 2.33, 2.27, $2.22\left(\mathrm{~s}, 1: 1: 2: 1: 1,6 \mathrm{Me}_{\mathrm{pz}}\right), 1.80\left(\mathrm{dt}, 1 \mathrm{H}, \mathrm{H}_{\mathrm{E}}\right)$.

${ }^{13} \mathbf{C}\left\{{ }^{1} \mathbf{H}\right\}$ NMR $\left(\mathrm{CDCl}_{3}, 25{ }^{\circ} \mathrm{C}\right): \delta 231.8\left(\mathrm{C}^{2}\right), 167.9(\mathrm{CO}), 150.8,150.7,150.7,143.9$, $143.7\left(1: 2: 1: 1: 1, \mathrm{C}_{\mathrm{qpz}}\right), 109.3,107.2,106.9\left(\mathrm{CH}_{\mathrm{pz}}\right), 46.13\left(\mathrm{C}^{3}\right), 14.4,14.3,14.1,13.0$, 12.5, $12.4\left(\mathrm{Me}_{\mathrm{pz}}\right), 11.8\left(\mathrm{C}^{1}\right),-15.3\left(\mathrm{C}^{4}\right)$.

Anal. Calc. for $\mathrm{C}_{20} \mathrm{H}_{28} \mathrm{~N}_{6} \mathrm{BO}_{2} \mathrm{Ir}$ : C, 40.9; H, 4.8; N, 14.3. Exp.: C, 40.6; H, 4.6; N, 14.0. 
Compound 1: To a cold $\left(0{ }^{\circ} \mathrm{C}\right)$ solution of $4(0.10 \mathrm{~g}, 0.17 \mathrm{mmol})$ in $\mathrm{C}_{6} \mathrm{H}_{6}(5 \mathrm{~mL})$, $\mathrm{HCO}_{2} \mathrm{Et}(0.18 \mathrm{~mL})$ and methanol $(0.1 \mathrm{~mL})$ were added. The resulting mixture was then added dropwise over $\mathrm{NaH}$ (0.06 g, oil free), stirred a room temperature for $48 \mathrm{~h}$ and added to cracked ice. The mixture was acidified with $\mathrm{HCl}\left(5 \mathrm{~mL}, 10 \%\right.$ in $\left.\mathrm{H}_{2} \mathrm{O}\right)$ and the organic layer separated. After extraction with $\mathrm{Et}_{2} \mathrm{O}(3 \times 5 \mathrm{~mL})$, the combined organic phases were dried with $\mathrm{Na}_{2} \mathrm{SO}_{4}$ and taken to dryness. The crude product was crystallized from $\mathrm{Et}_{2} \mathrm{O}: \mathrm{CH}_{2} \mathrm{Cl}_{2}(1: 2)$ at $-20{ }^{\circ} \mathrm{C}$. Isolated yield: $82 \%$.
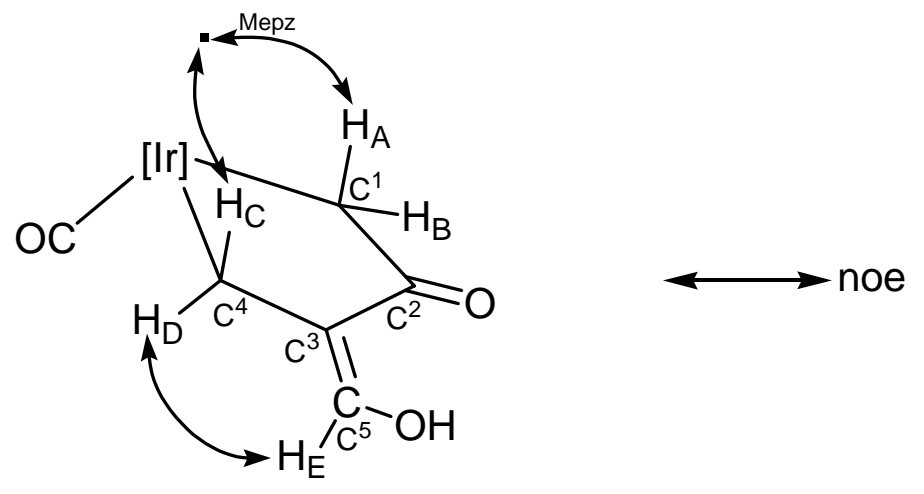

\section{Spectroscopic and analytical data}

IR (Nujol): v 2009 (Ir-CO), $1591 \mathrm{~cm}^{-1}(\mathrm{C}=\mathrm{O})$.

${ }^{1} \mathbf{H}$ NMR $\left(\mathrm{CDCl}_{3}, 25^{\circ} \mathrm{C}\right): \delta 8.58\left(\mathrm{~s}, 1 \mathrm{H}, \mathrm{H}_{\mathrm{E}}\right), 5.81,5.80,5.78\left(\mathrm{~s}, 1 \mathrm{H}\right.$ each, $\left.3 \mathrm{CH}_{\mathrm{pz}}\right)$, $3.25\left(\mathrm{~d}, 1 \mathrm{H},{ }^{2} J_{\mathrm{CD}}=12.9 \mathrm{~Hz}, \mathrm{H}_{\mathrm{D}}\right), 3.09\left(\mathrm{~d}, 1 \mathrm{H},{ }^{2} J_{\mathrm{AB}}=18.3 \mathrm{~Hz}, \mathrm{H}_{\mathrm{B}}\right), 2.98\left(\mathrm{~d}, 1 \mathrm{H}, \mathrm{H}_{\mathrm{A}}\right)$, $2.53\left(\mathrm{~d}, 1 \mathrm{H}, \mathrm{H}_{\mathrm{C}}\right), 2.39,2.34,2.32,2.14\left(\mathrm{~s}, 1: 3: 1: 1,6 \mathrm{Me}_{\mathrm{pz}}\right)$. The $\mathrm{OH}$ group has not been located.

${ }^{13} \mathbf{C}\left\{{ }^{1} \mathbf{H}\right\}$ NMR $\left(\mathrm{CDCl}_{3}, 25{ }^{\circ} \mathrm{C}\right): \delta 204.7\left(\mathrm{C}^{2}\right), 180.0\left(\mathrm{C}^{5},{ }^{1} J_{\mathrm{CH}}=174 \mathrm{~Hz}\right), 167.1(\mathrm{CO})$, $151.4,150.9,150.8,143.9,143.6\left(1: 1: 1: 1: 2, \mathrm{C}_{\mathrm{qpz}}\right), 121.2\left(\mathrm{C}^{3}\right), 109.1,107.2,107.0$ $\left(\mathrm{CH}_{\mathrm{pz}}\right), 14.3,14.2,12.9,12.7,12.5,12.4\left(\mathrm{Me}_{\mathrm{pz}}\right), 4.2\left(\mathrm{C}^{1},{ }^{1} J_{\mathrm{CH}}=129 \mathrm{~Hz}\right),-10.9\left(\mathrm{C}^{4},{ }^{1} J_{\mathrm{CH}}\right.$ $=131 \mathrm{~Hz})$. 
Compound 5: To a cold $\left(0{ }^{\circ} \mathrm{C}\right)$ solution of $1(0.10 \mathrm{~g}, 0.16 \mathrm{mmol})$ in $\mathrm{CH}_{2} \mathrm{Cl}_{2}(5 \mathrm{~mL})$, $\mathrm{TsN}_{3}(0.13 \mathrm{~g})$ and $\mathrm{NEt}_{3}(0.2 \mathrm{~mL})$ were added. The resulting mixture was stirred at room temperature for $20 \mathrm{~h}$ and then the volatiles were removed in vacuo. Compound 5 was isolated by chromatography on silica gel with hexane as eluent. Isolated yield: $46 \%$. This complex can be crystallized from hexane: $\mathrm{CH}_{2} \mathrm{Cl}_{2}(1: 2)$ at $-20^{\circ} \mathrm{C}$.<smiles>O=CC1CCCC1</smiles>

\section{Spectroscopic and analytical data}

IR (Nujol): v $2019(\mathrm{Ir}-\mathrm{CO}), 1692 \mathrm{~cm}^{-1}(\mathrm{C}=\mathrm{O})$.

${ }^{1} \mathbf{H}$ NMR $\left(\mathrm{CDCl}_{3}, 25{ }^{\circ} \mathrm{C}\right): \delta 6.48,5.24\left(\mathrm{~s}, 1 \mathrm{H}\right.$ each, $\left.\mathrm{C}^{4} \mathrm{H}_{2}\right), 5.88,5.84,5.68(\mathrm{~s}, 1 \mathrm{H}$ each, $\left.3 \mathrm{CH}_{\mathrm{pz}}\right), 2.98,2.72\left(\mathrm{~d}, 1 \mathrm{H}\right.$ each, $\left.{ }^{2} J_{\mathrm{HH}}=14.6 \mathrm{~Hz}, \mathrm{C}^{1} \mathrm{H}_{2}\right), 2.38,2.37,2.32,2.27,2.10$ (s, $\left.2: 1: 1: 1: 1,6 \mathrm{Me}_{\mathrm{pz}}\right)$.

${ }^{13} \mathbf{C}\left\{{ }^{1} \mathbf{H}\right\}$ NMR $\left(\mathrm{CDCl}_{3}, 25{ }^{\circ} \mathrm{C}\right): \delta 214.2\left(\mathrm{C}^{2}\right), 165.2(\mathrm{CO}), 152.1,151.4,150.7,144.1$, $144.0\left(1: 1: 1: 1: 2, C_{\mathrm{qpz}}\right), 130.7\left(\mathrm{C}^{3}\right), 120.3\left(\mathrm{C}^{4},{ }^{1} J_{\mathrm{CH}}=159\right.$ and $\left.156 \mathrm{~Hz}\right), 109.5,106.7$, $106.5\left(\mathrm{CH}_{\mathrm{pz}}\right), 14.4,14.3,14.1,13.1,12.4,12.3\left(\mathrm{Me}_{\mathrm{pz}}\right), 4.0\left(\mathrm{C}^{1},{ }^{1} J_{\mathrm{CH}}=136 \mathrm{~Hz}\right)$.

Anal. Calc. for $\mathrm{C}_{20} \mathrm{H}_{26} \mathrm{~N}_{6} \mathrm{BO}_{2} \mathrm{Ir}$ : C, 41.0; H, 4.4; N, 14.3. Exp: C, 41.6; H, 4.6; N, 13.7 . 
Compound 6: To a cold $\left(0{ }^{\circ} \mathrm{C}\right)$ solution of $5(0.10 \mathrm{~g}, 0.17 \mathrm{mmol})$ in $\mathrm{CH}_{2} \mathrm{Cl}_{2}(5 \mathrm{~mL})$, an excess of $m-\mathrm{ClC}_{6} \mathrm{H}_{4} \mathrm{CO}_{3} \mathrm{H}\left(0.13 \mathrm{~g}\right.$ dissolved in $5 \mathrm{~mL}$ of $\left.\mathrm{CH}_{2} \mathrm{Cl}_{2}\right)$ was added. After stirring at room temperature for $3 \mathrm{~h}$, the mixture was washed with $\mathrm{NaHCO}_{3}$-saturated water, the organic phase dried with $\mathrm{Na}_{2} \mathrm{SO}_{4}$ and taken to dryness. Compound $\mathbf{6}$ was purified by chromatography on silica gel with hexane: $\mathrm{Et}_{2} \mathrm{O}(2: 1)$ as eluent. Isolated yield: $60 \%$.
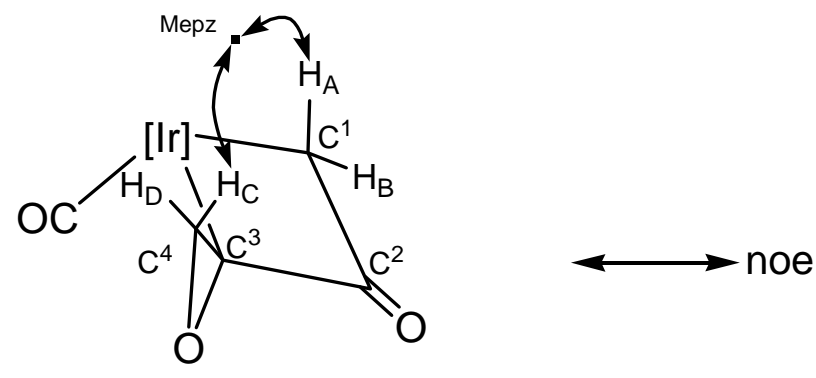

\section{Spectroscopic and analytical data}

IR (Nujol): v $2023(\mathrm{Ir}-\mathrm{CO}), 1714 \mathrm{~cm}^{-1}(\mathrm{C}=\mathrm{O})$.

${ }^{1} \mathbf{H}$ NMR $\left(\mathrm{CDCl}_{3}, 25^{\circ} \mathrm{C}\right): \delta 5.85,5.79,5.76\left(\mathrm{~s}, 1 \mathrm{H}\right.$ each, $\left.3 \mathrm{CH}_{\mathrm{pz}}\right), 3.10\left(\mathrm{~d}, 1 \mathrm{H},{ }^{2} J_{\mathrm{BA}}=\right.$ $\left.14.6 \mathrm{~Hz}, \mathrm{H}_{\mathrm{A}}\right), 3.05\left(\mathrm{~d}, 1 \mathrm{H},{ }^{2} J_{\mathrm{DC}}=6.6 \mathrm{~Hz}, \mathrm{H}_{\mathrm{C}}\right), 2.86\left(\mathrm{~d}, 1 \mathrm{H}, \mathrm{H}_{\mathrm{D}}\right), 2.82\left(\mathrm{~d}, 1 \mathrm{H}, \mathrm{H}_{\mathrm{B}}\right)$, $2.37,2.36,2.35,2.30,2.28,2.16$ (s, $3 \mathrm{H}$ each, $6 \mathrm{Me}_{\mathrm{pz}}$ ).

${ }^{13} \mathbf{C}\left\{{ }^{1} \mathbf{H}\right\}$ NMR $\left(\mathrm{CDCl}_{3}, 25{ }^{\circ} \mathrm{C}\right): \delta 217.2\left(\mathrm{C}^{2}\right), 164.4(\mathrm{CO}), 152.2,151.2,144.4,143.9$ $\left(1: 2: 1: 2, \mathrm{C}_{\mathrm{qpz}}\right), 109.7,106.8,106.7\left(\mathrm{CH}_{\mathrm{pz}}\right), 53.3\left(\mathrm{C}^{4},{ }^{1} J_{\mathrm{CH}}=177\right.$ and $\left.174 \mathrm{~Hz}\right), 41.0\left(\mathrm{C}^{3}\right)$, $14.5,14.0,13.1,13.0,12.4,12.3\left(\mathrm{Me}_{\mathrm{pz}}\right), 1.0\left(\mathrm{C}^{1},{ }^{1} J_{\mathrm{CH}}=139 \mathrm{~Hz}\right)$. 
Compound 7: Through a solution of complex $5(0.10 \mathrm{~g}, 0.17 \mathrm{mmol})$ in $\mathrm{CH}_{2} \mathrm{Cl}_{2}(20$ $\mathrm{mL})$, at $-70^{\circ} \mathrm{C}, \mathrm{O}_{3}$ was bubbled for $10 \mathrm{~min}$. Then $\mathrm{Me}_{2} \mathrm{~S}(0.05 \mathrm{~mL})$ was added and the mixture stirred at room temperature for $3 \mathrm{~h}$. After washing with water, the organic phase was dried with $\mathrm{Na}_{2} \mathrm{SO}_{4}$ and the volatiles removed in vacuo. Compound 7 was purified by chromatography on silica gel with hexane: $\mathrm{Et}_{2} \mathrm{O}(10: 1)$ as eluent. Isolated yield: $56 \%$.<smiles>O=C1C[Al]2CCCC1O2</smiles>

Spectroscopic and analytical data

IR (Nujol): v $2033(\mathrm{Ir}-\mathrm{CO}), 1710 \mathrm{~cm}^{-1}(\mathrm{C}=\mathrm{O})$.

${ }^{1} \mathbf{H}$ NMR $\left(\mathrm{CDCl}_{3}, 25^{\circ} \mathrm{C}\right): \delta 5.89,5.83,5.72\left(\mathrm{~s}, 1 \mathrm{H}\right.$ each, $\left.3 \mathrm{CH}_{\mathrm{pz}}\right), 2.73,2.71(\mathrm{AB}$ spin system, $\left.2 \mathrm{H},{ }^{2} J_{\mathrm{HH}}=14.0 \mathrm{~Hz}, \mathrm{C}^{1} \mathrm{H}_{2}\right), 2.41,2.39,2.37,2.33,2.32,2.23$ (s, $3 \mathrm{H}$ each, 6 $\left.\mathrm{Me}_{\mathrm{pz}}\right)$.

${ }^{13} \mathbf{C}\left\{{ }^{1} \mathbf{H}\right\}$ NMR $\left(\mathrm{CDCl}_{3}, 25{ }^{\circ} \mathrm{C}\right): \delta 188.6\left(\mathrm{C}^{2}\right), 160.5(\mathrm{CO}), 152.5,152.1,151.5,144.9$, 144.3, $144.1\left(\mathrm{C}_{\mathrm{qpz}}\right), 109.6,107.4,106.5\left(\mathrm{CH}_{\mathrm{pz}}\right), 14.9,13.5,12.9,12.7,12.6,12.0\left(\mathrm{Me}_{\mathrm{pz}}\right)$, $-6.1\left(\mathrm{C}^{1},{ }^{1} J_{\mathrm{CH}}=140 \mathrm{~Hz}\right)$.

Anal. Calc. for $\mathrm{C}_{20} \mathrm{H}_{26} \mathrm{~N}_{6} \mathrm{BO}_{2} \mathrm{Ir} \cdot \mathrm{C}_{6} \mathrm{H}_{14}$ : C, 43.6; H, 4.7; N, 12.7. Exp: C, 43.3; H, 4.4; N, 12.6 . 
X-Ray structure determination for compounds 5, 6 and 7: A single crystal of suitable size, coated with dry perfluoropolyether was mounted on a glass fiber and fixed in a cold nitrogen stream $[T=100(2) \mathrm{K}]$ to the goniometer head. Data collection was performed on Bruker-Nonius X8APEX-II CCD diffractometer, using monochromatic radiation $\lambda\left(\mathrm{Mo} \mathrm{K}_{\alpha}\right)=0.71073 \AA$, by means of $\omega$ and $\varphi$ scans with a width of 0.30 and an exposure times of $10 \mathrm{~s}$ (in the range $3.58^{\circ}<2 \theta<59.32^{\circ}, 5$ ) or $30 \mathrm{~s}$ (in the range $3.98^{\circ}<$ $2 \theta<51.20^{\circ}, \mathbf{6}$ and $\left.6.12^{\circ}<2 \theta<31.04^{\circ}, 7\right)$ per frame, with a detector distance of 37.5 $\mathrm{mm}$. The data were reduced (SAINT) ${ }^{1}$ and corrected for Lorentz polarization effects and absorption by multiscan method applied by $\mathrm{SADABS}^{2}$. The structure was solved by direct methods (SIR-2002) $)^{3}$ and refined against all $F^{2}$ data by full-matrix least-squares techniques (SHELXTL-6.12) ${ }^{4}$. All the non-hydrogen atoms were refined with anisotropic displacement parameters. The hydrogen atoms were included from calculated positions and refined riding on their respective carbon atoms with isotropic displacement parameters. (5): $\mathrm{C}_{20} \mathrm{H}_{26} \mathrm{BIrN}_{6} \mathrm{O}_{2}, M_{r}=585.48$, colourless block crystal $\left(0.46 \times 0.32 \times 0.22 \mathrm{~mm}^{3}\right)$ from hexane- $\mathrm{CH}_{2} \mathrm{Cl}_{2} ;$ monoclinic, space group $P 2_{1} / n$ (no. 14$)$, $a=8.0966(6) \AA, b=19.5568(13) \AA, c=14.0294(10) \AA, \beta=94.738(2)^{\circ}, V=2213.9(3)$ $\AA^{3}, Z=4, \rho_{\text {calcd }}=1.757 \mathrm{gcm}^{-3}, \mathrm{~F}(000)=1144, \mu=6.059 \mathrm{~mm}^{-1} .20959$ measured reflections, of which 6187 were unique $\left(\mathrm{R}_{\text {int }}=0.0420\right) ; 277$ refined parameters, final $R_{1}$ $=0.0679$, for reflections with $I>2 \sigma(I), w R_{2}=0.1645$ (all data), GOF $=1.027, \max$ difference peak and hole $=0.965$ and $-0.738 \mathrm{e} / \AA^{3}$. (6): $\mathrm{C}_{20} \mathrm{H}_{26} \mathrm{BIrN}_{6} \mathrm{O}_{3}, M_{r}=601.48$, colourless needle crystal $\left(0.17 \times 0.12 \times 0.09 \mathrm{~mm}^{3}\right)$ from hexane- $\mathrm{CH}_{2} \mathrm{Cl}_{2}$; orthorhombic, space group Pnma (no. 62), $a=20.463(2) \AA, b=13.6577(16) \AA, c=7.9547(9) \AA, \alpha=$ $\beta=\gamma=90^{\circ}, V=2223.2(4) \AA^{3}, Z=4, \rho_{\text {calcd }}=1.797 \mathrm{gcm}^{-3}, \mathrm{~F}(000)=1176, \mu=6.040$ $\mathrm{mm}^{-1} .12462$ measured reflections, of which 1997 were unique $\left(\mathrm{R}_{\mathrm{int}}=0.0583\right) ; 185$ refined parameters, final $R_{1}=0.0677$ for reflections with $I>2 \sigma(I), w R_{2}=0.1298$ (all 
data), GOF $=1.067$, max difference peak and hole $=1.605$ and $-3.790 \mathrm{e} / \AA^{3}$. The crystal structure presents pseudosymmetry through a mirror plane due to a positional disorder (in the same proportion) of two Iridium complex enantiomers. (7): $\mathrm{C}_{25} \mathrm{H}_{32} \mathrm{BIrN}_{6} \mathrm{O}_{3}$, $\left[\mathrm{C}_{18} \mathrm{H}_{24} \mathrm{BIrN}_{6} \mathrm{O}_{3}+\mathrm{C}_{7} \mathrm{H}_{8}\right.$ of a molecule of crystallization of toluene ], $M_{r}=667.58$, colourless plate crystal $\left(0.14 \times 0.11 \times 0.08 \mathrm{~mm}^{3}\right)$ from hexane- $\mathrm{CH}_{2} \mathrm{Cl}_{2}$; monoclinic, space group $P 2_{1}$ (no. 4), $a=9.8412(2) \AA, b=17.0571(3) \AA, c=15.9482(4) \AA, \beta=$ $99.1630(10)^{\circ}, V=2642.94(10) \AA^{3}, Z=4, \rho_{\text {calcd }}=1.678 \mathrm{gcm}^{-3}, \mathrm{~F}(000)=1320, \mu=$ $5.090 \mathrm{~mm}^{-1} .29102$ measured reflections, of which 14564 were unique $\left(R_{\text {int }}=0.0410\right)$; 650 refined parameters, final $R_{1}=0.0462$ for reflections with $I>2 \sigma(I), w R_{2}=0.1062$ (all data), GOF $=1.010$, max difference peak and hole $=4.659$ and $-2.192 \mathrm{e} / \AA^{3}$.

As pointed out by a reviewer, the $R_{1}$ values for $\mathbf{5}$ and $\mathbf{6}$ are unusually high. In the case of 5, this is due, in part to the poor quality of the crystal, and in part to twinning shown by reflections that should be absent in the space group $\mathrm{P} 2{ }_{1} / \mathrm{n}(\mathrm{h} 00(\mathrm{~h} \neq 2 \mathrm{n}), \mathrm{h} 01$ ( $h$ $+1 \neq 2 n)$, and $001(1 \neq 2 n))$. In this way the crystal appears to be disordered in a second domain rotated by $180^{\circ}$ about the direction [010]. Although these anomalous reflections were removed from the data set, the twinning affects the $F$ values $(|F c|$ slightly greater than $\left|F_{O}\right|$ ) and then the value of $R_{1}$. For the case of $\mathbf{6}$, the crystal structure presents pseudosymmetry, due to a positional disorder of the two enantiomers of the complex in the same proportion. In fact there is a reflection plane (010) which contains one dimethyl pyrazolyl ring, the Ir atom and the CO ligand, and is perpendicular to the epoxyketone ring. After refinement, the correct space group turned out to be the centrosymmetric Pnma, with higher symmetry than the non centrosymmetric Pna $2_{1}$. Again this disorder ( $\left|F_{c}\right|$ slightly greater of $\left.\left|F_{o}\right|\right)$ causes the unusually high $R_{1}$ value.

(1) SAINT 6.02, BRUKER-AXS, Inc., Madison, WI 53711-5373 USA, 1997-1999. 
(2) SADABS George Sheldrick, Bruker AXS, Inc., Madison, Wisconsin, USA, 1999.

(3) Burla, M. C.; Camalli, M.; Carrozzini, B.; Cascarano, G. L.; Giacovazzo, C.; Polidori, G.; Spagna, R. SIR2002: the program; J. Appl. Cryst. 2003, 36, 1103.

(4) SHELXTL 6.14, Bruker AXS, Inc., Madison, Wisconsin, USA, 2000-2003. 